Universidade de São Paulo

Escola Superior de Agricultura "Luiz de Queiroz"

Avaliação do potencial antimicrobiano do licor pirolenhoso de Dendrocalamus asper (Schult \& Schult) Backer

\title{
Luciana Jankowsky
}

Tese apresentada para obtenção do título de Doutora em Ciências, Programa: Recursos Florestais. Opção em: Tecnologia de Produtos Florestais

Piracicaba

2019 


\section{Luciana Jankowsky}

Farmacêutica

Avaliação do potencial antimicrobiano do licor pirolenhoso de Dendrocalamus asper (Schult \& Schult) Backer

versão revisada de acordo com a resolução CoPGr 6018 de 2011

Orientador:

Prof. Dr. JOSÉ OTÁVIO BRITO

Tese apresentada para obtenção do título Doutora em Ciências, Programa: Recursos Florestais. Opção em: Tecnologia de Produtos Florestais 
Dados Internacionais de Catalogação na Publicação DIVISÃO DE BIBLIOTECA - DIBD/ESALQ/USP

Jankowsky, Luciana

Avaliação do potencial antimicrobiano do licor pirolenhoso de Dendrocalamus asper (Schult \& Schult) Backer / Luciana Jankowsky. versão revisada de acordo com a resolução CoPGr 6018 de 2011. -Piracicaba, 2019.

$126 \mathrm{p}$.

Tese (Doutorado) - - USP / Escola Superior de Agricultura "Luiz de Queiroz".

1. Cromatografia gasosa 2. Microscopia eletrônica de varredura 3. Escherichia coli 4. Staphylococcus aureus I. Título 
RESUMO

Avaliação do potencial antimicrobiano do licor pirolenhoso de Dendrocalamus asper

(Schult \& Schult) Backer

Considerando que o licor pirolenhoso obtido de diferentes tipos de biomassa possui atividades antivirais, o principal objetivo da presente pesquisa foi avaliar a ação antimicrobiana do licor pirolenhoso obtido na pirólise do bambu (Dendrocalamus asper), e de suas frações metanólica e hexânica, em relação às bactérias Escherichia coli e Staphylococcus aureus. O licor pirolenhoso foi recuperado da pirólise em mufla laboratorial, nas temperaturas de $250^{\circ} \mathrm{C}, 350^{\circ} \mathrm{C}$ e $550^{\circ} \mathrm{C}$, os quais foram submetidos a separação líquido-líquido com metanol e hexano gerando as frações metanólica e hexânica. Os licores pirolenhosos integrais (na dose de 5,0 mg por disco) e suas respectivas frações metanólica e hexânica (na dose de $0,125 \mathrm{mg}$ por disco), foram aplicadas no ensaio de difusão em disco com as bactérias $E$. coli e $S$. aureus; sendo que nesse ensaio a fração metanólica do licor obtido a $350^{\circ} \mathrm{C}(\mathrm{FMeOH} 350)$ apresentou melhor eficiência e consistência nos resultados e foi selecionada para continuidade da pesquisa. Através da análise por cromatografia gasosa acoplada a espectrometria de massa foi possível identificar qualitativamente 92 compostos químicos na fração FMeOH350. Essa fração foi aplicada no ensaio de difusão com as bactérias $E$. coli e $S$. aureus nas doses de $0,125 \mathrm{mg}, 0,500 \mathrm{mg}, 0,750 \mathrm{mg}$ e $1,000 \mathrm{mg}$. O tratamento com a fração metanólica obtida a $350^{\circ} \mathrm{C}$ inibiu o crescimento celular das bactérias, na região ao redor da aplicação das amostras (halo de inibição), e causou uma variedade de deformações morfológicas em tamanho e deformidades nas paredes celulares das bactérias, comprovadas por aplicação da microscopia eletrônica de varredura, estabelecendo um perfil de atividade antimicrobiana. Esses resultados estão em acordo com a Política Nacional de Incentivos ao Cultivo e Manejo Sustentável do Bambu, bem como traz benefícios para a sociedade e o ambiente como um todo, demonstrando a possibilidade de agregar valor a um subproduto da pirólise.

Palavras-chave: Cromatofia gasosa; Microscopia eletrônica de varredura; Escherichia coli; Staphylococcus aureus 


\begin{abstract}
Antimicrobial activity evaluation of Dendrocalamus asper (Schult \& Schult) Backer

pyroligneous liquor
\end{abstract}

Considering that the pyroligneous liquor obtained from various types of biomass has antiviral activity, the main objective of the present study was to evaluate the antimicrobial action of the pyroligneous liquor obtained in the pyrolysis of bamboo (Dendrocalamus asper) and its methanolic and hexanic fractions, in relation to Escherichia coli and Staphylococcus aureus bacteria. The pyroligneous liquor was recovered by laboratorial bamboo pyrolysis at temperatures of $250^{\circ} \mathrm{C}$, $350^{\circ} \mathrm{C}$ and $550^{\circ} \mathrm{C}$, which were then fractioned by liquid-liquid separation with methanol and hexane. The three integral pyroligneous liquor (dosage of 5,0 mg per disc) and its respective methanolic and hexanic fractions (dosage of 0,125 mg per disc) were used in the diffusion disc assay of $E$. coli and $S$. aureus; and the result was the methanolic fraction obtained at $350^{\circ} \mathrm{C}$ (MeOHF350) showing the better efficiency and results consistence, being selected for the research continuity. Through gas chromatography coupled to mass spectrometry it was possible to qualitatively identify 92 chemical compounds in this methanolic fraction. The MeOHF350 fraction was used in the diffusion disc assay of $E$. coli and $S$. aureus at dosages of $0.125,0.500,0.750$ and $1.000 \mathrm{mg}$. Treatment with the methanolic fraction obtained at $350^{\circ} \mathrm{C}$ inhibited cellular growth, in the region around of samples application (inhibition halo), caused a variety of morphological variations in size as well as deformities in the bacterial cell walls, proved through scanning electron microscopy, establishing an antimicrobial activity profile. This finding supports the National Policy for Incentives for Sustainable Management and Cultivation of Bamboo (PNMCB) and has benefits to the society and environment as a whole, demonstrating the possibility of adding value to a byproduct of pyrolysis.

Keywords: Gas chromatography; Scanning electron microscopy; Escherichia coli; Staphylococcus aureus 


\section{INTRODUÇÃO}

O Brasil possui, em seu território, um bioma constituído por tipos de vegetação contínuas e identificáveis a nível regional, relacionados de acordo as características geoclimáticas e agrupamentos de vida vegetal e animal em ambientes similares, favorecendo uma biodiversidade complexa (SNIF, 2016). As figuras 1 e 2 ilustram este panorama.

\begin{tabular}{lcc|}
\hline & Área dos biomas do Brasil \\
& Área aproximada $\left(\mathrm{em} \mathrm{km}^{2}\right)$ & $\%$ Brasil \\
Amazônia & 4.196 .943 & 49,29 \\
Cerrado & 2.036 .448 & 23,92 \\
Mata Atlântica & 1.110 .182 & 13,04 \\
Caatinga & 844.453 & 9,92 \\
Pampa & 176.496 & 2,07 \\
Pantanal & 150.355 & 1,76 \\
Total & $\mathbf{8 . 5 1 4 . 8 7 7}$ & $\mathbf{1 0 0}$ \\
\hline
\end{tabular}

Figura 1. Áreas de biomas do Brasil, segundo o IBGE em 2009 (SNIF, 2016).

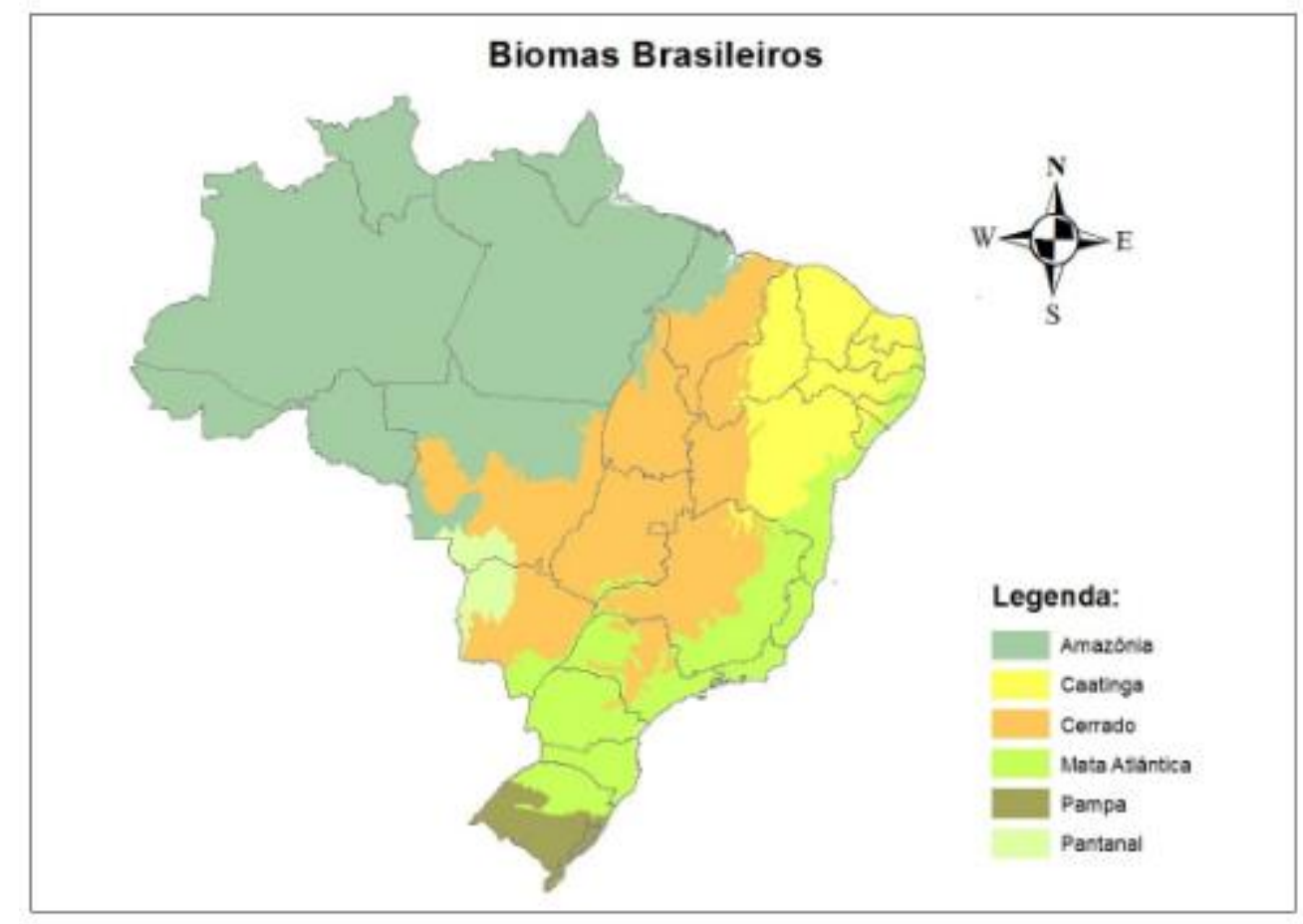

Figura 2. Mapa dos biomas do Brasil, de acordo com o IBGE (SNIF, 2016).

As biomassas, decorrentes da diversidade de biomas brasileiros, são fonte de recursos renováveis, considerados sustentáveis quando comparados aos recursos fósseis, cuja diversidade, disponibilidade e valor energético fornecem energia de inúmeras maneiras, tais como combustíveis sólidos, líquidos ou gases, assim como calor e eletricidade. Devido a 
estas características as biomassas são consideradas uma opção à fontes de energia oriundas de fósseis (DEMIRBAS, 2009; MENA, 2014; RABELO, 2018).

Considera-se que a biodiversidade brasileira possui uma enorme gama de compostos com complexidade e variedade de estruturas moleculares, as quais representam uma fonte com potencial de pesquisa e inovação (DA SILVA; RODRIGUES, 2014). Os compostos químicos presentes no bioma vegetal, passíveis de serem utilizados em diversos segmentos das industrias químicas, farmacêuticas e alimentícias, são oriundos do metabolismo segundário da planta, seja de origem madeireira ou não madeireira. Diversos processos podem ser utilizados para a extração, de maneira a serem empregados para o consumo humano ou animal (JANKOWSKY et al., 2017).

Dentro da biodiversidade brasileira deve-se considerar a importância das diversas espécies de bambu, nativas ou introduzidas, visto que várias dessas espécies apresentam taxas de crescimento e formação de biomassa comparáveis às obtidas em plantios homogêneos de espécies florestais. Dentre as de interêsse econômico destacam-se o Dendrocalamus asper, o Dendrocalamus giganteus, o Dendrocalamus latiflorus e o Bambusa vulgaris; sendo que este último tem sido especificamente usado na produção de carvão vegetal, celulose e papel (GRECO; CROMBERG, 2011; GUARNETTI, 2013).

O processo mais amplamente empregado para a conversão da biomassa vegetal em carvão é a pirólise, com aplicação direta na conversão e utilização de calor. No decorrer deste processo diversas faixas de temperatura são aplicadas à biomassa, originando a liberação de gases não-condensáveis e condensáveis; sendo estes útimos denominados licor pirolenhoso, ou extrato pirolenhoso (DIAS et al., 2018). Muitos estudos já foram realizados para se determinar a composição química do licor pirolenhoso, considerando espécies vegetais específicas, assim como sua atividade biológica, configurando uma abordagem sustentável e de "química verde". O bambu pode ser inserido nesse contexto e, visando contribuir para a ampliação de conhecimentos nesse campo, foi conduzida a presente pesquisa. O estudo focou a espécie Dendrocalamus asper considerando a conversão da biomassa em carvão vegetal e, especificamente; o potencial de uso de compostos presentes no licor pirolenhoso com propriedades passíveis de serem empregados como conservantes. 


\section{CONCLUSÕES}

A realização do estudo com o licor pirolenhoso oriundo da espécie de bambu Dendrocalamus asper (Schulther) Backer demonstrou que:

I. Os rendimentos dos licores pirolenhosos obtidos nas temperaturas de $250^{\circ} \mathrm{C}$, $350^{\circ} \mathrm{C}$ e $550^{\circ} \mathrm{C}$ são coerentes entre si, pois quanto maior a temperatura empregada para degradar o bambu em carvão vegetal, maior a quantidade de licor pirolenhoso oriundo do processo.

II. Os licores pirolenhosos integrais são compostos majoritariamente por água, e nesta estão diluídos os compostos químicos.

III. Através do fracionamento líquido-líquido de cada licor pirolenhoso integral, nas diferentes temperaturas de pirólise $\left(250^{\circ} \mathrm{C}, 350^{\circ} \mathrm{C}\right.$ e $\left.550^{\circ} \mathrm{C}\right)$, foi possível obter frações de diferentes polaridades (FMeOH e FHex), respectivamente. O fracionamento permitiu a separação dos compostos químicos por afinidade a polaridade do solvente extrator. Esta análise foi confirmada por cromatografia de camada delgada.

IV. Através do teste de difusão de disco foi confirmada o potencial antimicrobiano dos licores pirolenhosos integrais e suas respectivas frações, para as bactérias Escherichia coli e Staphylococcus aureus, através da análise de inibição de halo.

V. Os dados de rendimento, perfil químico encontrado na análise de CCD e a atividade biológica permitiram selecionar a fração metanólica FMeOH350 para estudos de cromatografia gasosa acoplada a espectômetro de massas, microscopia eletrônica de varredura e análise do perfil antimicrobiano, com potencial aplicabilidade em sistemas conservantes.

VI. A composição química da $\mathrm{FMeOH} 350$ é complexa, apresentando 92 compostos diferentes. Os fenóis, os catecois e o ácido acético são os compostos mais relacionados na literatura com atividade antimicrobiana.

VII. As análises de imagem obtidas por microscopia eletrônica de varredura evidenciam as alterações que a FMeOH350 promove na parede celular de ambas Escherichia coli e Staphylococcus aureus, apesar da sua diferença química e estrutural (Gram negativa e Gram positiva, respectivamente). 
VIII. A curva dose-resposta da FMeOH350 é dose-dependente, tendo aplicabilidade em sistemas conservantes em diversas áreas, tais como farmacêutica, química, cosmética, veterinária e alimentícia. 


\section{REFERÊNCIAS}

ABERNETHY, J. et al. Epidemiology of Escherichia coli bacteraemia in England: results of an enhanced sentinel surveillance programme. Journal of Hospital Infection, v. 95, n. 4, p. 365-375, 2017.

AGÊNCIA NACIONAL DE VIGILâNCIA SANITÁRIA (ANVISA). Padronização dos Testes de Sensibilidade a Antimicrobianos por Disco-difusão: Norma Aprovada - Oitava Edição. Clinical and Laboratory Standards Institute - CLSI, v. 23, n. 1, p. 1-58, 2003.

AKIRA, A. et al. Bambu - Dossiê Técnico. Intituto de Tecnologia do Paraná - TECPAR, p. 1-2, Ago. 2007. ALMEIDA, R. Potencial do licor pirolenhoso da madeira de eucalipto como agente conservante de cosméticos e saneantes. [s.1.] Escola Superior de Agricultura "Luiz de Queiroz" - Universidade de São Paulo, 2012.

ALSALH, I M. S. et al. Green synthesis of silver nanoparticles using Pimpinella anisum seeds: Antimicrobial activity and cytotoxicity on human neonatal skin stromal cells and colon cancer cells. International Journal of Nanomedicine, v. 11, p. 4439-4449, 2016.

ANVISA. Resolução $\mathbf{N}^{\circ} 79$ de 25 de agosto de 2000Agência Nacional de Vigilância SanitáriaBrasilMinistério da Saúde, , 2010. Disponível em: <http://www.arca.fiocruz.br/handle/icict/13356>

BALAT, M. et al. Main routes for the thermo-conversion of biomass into fuels and chemicals. Part 1: Pyrolysis systems. Energy Conversion and Management, v. 50, n. 12, p. 3147-3157, 2009.

BERnADInO, C. A. R. Obtenção do Carvão de Pirólise a partir da Torta de Filtro Proveniente de Usinas Sucroalcooleiras. [s.1.] Universidade Federal do Rio de Janeiro, 2014.

BRAGE, C.; SJÖSTRÖM, K. Separation of phenols and aromatic hydrocarbons from biomass tar using aminopropylsilane normal-phase liquid chromatography. Journal of Chromatography A, v. 538, n. 2, p. 303$310,1991$.

BRASIL. LEI No 12.484, DE 8 DE SETEMBRO DE 2011. Disponível em: <http://www.planalto.gov.br/ccivil_03/_Ato2011-2014/2011/Lei/L12484.htm>.

BRITO, F. M. S. Efeito da Termorretificação nas Propriedades Tecnológicas do Bambu. [s.1.] Universidade do Espírito Santo, 2013.

BRITO, F. M. S. Produção e avaliação da qualidade de painéis aglomerados constituídos por partículas de bagaço de cana-de-açúcar e bambu Piracicaba 2018. [s.1.] Universidade de São Paulo - Usp, 2018.

BRITO, J. O. Princípios de Produção e Utilização de Carvão Vegetal de Madeira. Documentos Florestas, n. 9, p. $1-19,1990$.

BRITO, J. O.; TOMAZELLO FILHO, M.; SALGADO, A. L. DE B. Produção e caracterização do carvão vegetal de espécies e variedades de bambu. Ipef, ..., v. 36, p. 13-17, 1987.

BUDYKO, M. I. Climate prediction based on past and current analogues. Climate change. Proc. 2nd World Climate Conference, Geneva, 1990, v. 103, n. May, p. 225-229, 1991.

CAI, J. et al. Review of Physicochemical Properties and Analytical Characterization of Lignocellulosic Biomass. Renewable and Sustainable Energy Reviews, v. 76, p. 309-322, 2017.

CAMPOS, Â. D. Técnicas para Produção de Extrato Pirolenhoso para Uso Agricola. Circular Técnica EMBRAPA, n. 65, p. 1-8, 2007.

CAMPOS, J. V. DE. Avaliação da atividade antimicrobiana e análise morfológica por microscopia de força 
atômica (AFM) da ação de extratos de própolisverde sobre Staphylococcus aureus e Escherichia coli. [s.1.] Universidade Federal de São Carlos, 2017a.

CAMPOS, J. V. Avaliação da atividade antimicrobiana e análise morfológica por microscopia de força atômica (AFM) da ação de extratos de própolis verde sobre Staphylococcus aureus e Escherichia coli. [s.l.] Universidade Federal de São Carlos, 2017b.

CARTAXO, R. T. Fracionamento bioguiado e caracterização química de composto com atividade antimicrobiana de geoprópolis de Uruçu Nordestina: abelha indígena sem ferrão Melipona scutellaris. [s.l.] Universidade Federal de Alfenas, 2012.

CARVALHO, A. S. Estudo Prospectivo de Colonização por Staphylococcus aureus Resistente à Meticilina um Serviço de Medicina Interna: População, Factores de Risco e Implicações. Medicina Interna, v. 24, n. 3, p. $208-$ 216, 2017.

CHALLAGUNDLA, L. et al. Range Expansion and the Origin of USA300 North American Epidemic MethicillinResistant Staphylococcus aureus. mBio, v. 8, n. 1, p. e02016-17, 2018.

COLYE, M. S. et al. Manual of antimicrogial Susceptibility Testing. (M. B. Coyle, Ed.)Journal of Experimental Psychology: General. Anais...American Society for Microbiology, 2005

Conservantes. Cosméticos e Conservantes, v. 44, p. 25, 2011.

CORRÊA, F. A. F. Características dos patótipos de E . coli e implicações de E . coli patogênica para aves em achados de abatedouros frigoríficos. [s.l.] Universidade Federal de Goiás, 2012.

COSTA, R. DE S. M. O Bambu: potencialidades técnicas e sociais e a políticas de incentivo ao manejo sustentado. [s.1.] Universidade Federal Fluminense, 2014.

Cromatografia de Camada Delgada - CCD. São PauloInstituto de Química - USP, , 2013. Disponível em: <http://www.iq.usp.br/wjbaader/qfl2343/Coloquio_CCD_2013.pdf>

DA COSTA SÃO PEDRO, T.; MORCILLO, A. M.; BARACAT, E. C. E. Etiology and prognostic factors of sepsis among children and adolescents admitted to the intensive care unit. Revista Brasileira de Terapia Intensiva, v. 27, n. 3, p. 240-246, 2015.

DA SILVA, V. C.; RODRIGUES, C. M. Natural products: an extraordinary source of value-added compounds from diverse biomasses in Brazil. Chemical and Biological Technologies in Agriculture, v. 1, n. 1, p. 1-6, 2014. DAZZI, A. et al. AFM-IR: Combining atomic force microscopy and infrared spectroscopy for nanoscale chemical characterization. Applied Spectroscopy, v. 66, n. 12, p. 1365-1384, 2012.

DE BRITO, J. S. Utilização do broto de bambu como possibilidade de diversificação alimentar no município de Taquara/RS. [s.1.] Faculdade de Ciências Econômicas da UFRGS, 2013.

DE OLIVEIRA SOUZA, C. et al. Escherichia coli enteropatogênica: uma categoria diarreiogênica versátil. Revista Pan-Amazônica de Saúde, v. 2, n. 7, p. 1-2, 2016.

DEMIRBAS, A. Reuse of wood wastes for energy generation. Energy Sources, Part A: Recovery, Utilization and Environmental Effects, v. 31, n. 19, p. 1687-1693, 2009.

DIAS, A. F. et al. Pyrolysis and wood by-products of species from the Brazilian semi-arid region. Scientia Forestalis/Forest Sciences, v. 46, n. 117, 2018.

DOS REIS, G. V. Isolamento bioguiado de compostos de actinobactérias com atividade fungitóxica. [s.l.] Escola Superior de Agricultura «Luiz de Queiroz», Universidade de São Paulo (ESALQ/USP), 2017.

DUNCAN, T. V. Applications of nanotechnology in food packaging and food safety: Barrier materials, 
antimicrobials and sensors. Journal of Colloid and Interface Science, v. 363, n. 1, p. 1-24, 2011.

EPAND, R. M. et al. Molecular mechanisms of membrane targeting antibiotics 论. Biochimica et Biophysica Acta, v. 1858, n. 5, p. 980-987, 2016.

EVANS JR, D. J.; EVANS, D. . G. Escherichia coli in diarrheal disease. Em: Medical Microbiology. [s.l: s.n.]. p. Chapter 25.

FANI, M. Conservação de alimentos por aditivos químicos. Food Ingredients Brasil, v. XIV, n. 22, p. 35-42, 2012.

FELISBERTO, M. H. F. et al. Young bamboo culm: Potential food as source of fiber and starch. Food Research International, v. 101, n. September, p. 96-102, 2017.

FERREIRA, F. DE S. Aditivos Alimentares E Suas Reações Adversas No Consumo. Revista da Universidade Vale do Rio Verde, v. 13, n. 1, p. 397-407, 2015.

FERREIRA, L. F. B.; SARAIVA, L. M.; PEDROSO, N. Cromatografia em Camada DelgadaAraraquaraUNESP, , 2018.

FOSTER, T. Staphylococcus. Em: BARON, S. (Ed.). . Medical Microbiolog. 4 edt. ed. Galviston: University of Texas Medical Branch at Galveston;, 1996.

GALVÃO, A. P. M.; JANKOWSKY, I. P. Secagem Racional de Madeira. 1 ed ed. São Paulo: NObel, 1985.

GHAVAMI, K. Bamboo as reinforcement in structural concrete elements. Cement and Concrete Composites, v. 27, n. 6, p. 637-649, 2005.

GOMES, A. B. Alergia a cosméticos. Aditivos Cosméticos, Mar. 2017.

GRECO, T. M.; CROMBERG, M. Bumbu: cultivo e manejo. Florianópolis: Insular, 2011.

GUARNETTI, R. L. Cogeração De Eletricidade Utilizando Bambu No Brasil: Aspectos Técnicos Econômicos e Ambientais. [s.1.] Falculdade de Economia e Administração-Instituto de Energia e Ambiente, 2013.

GUENTZEL, M. N. Escherichia, Klebsiella, Enterobacter, Serratia, Citrobacter, and Proteus. Em: BARON, S. (Ed.). . Medical Microbiology. 4 edt. ed. Galveston (TX): Medical Microbiology, 1996.

HADI, S.; BREMNER, J. B. Initial studies on alkaloids from Lombok medicinal plants. Molecules, v. 6, n. 2, p. 117-129, 2001.

HARRIS, L. G. et al. An introduction to Staphylococcus aureus, and techniques for identifyingand quantifying S. aureus adhesins in relation to adhesion to biomaterials:Review. European Cells and Materials, v. 4, p. 39-60, 2002.

HARTMANN, M. et al. Damage of the bacterial cell envelope by antimicrobial peptides gramicidin S and PGLa as revealed by transmission and scanning electron microscopy. Antimicrobial Agents and Chemotherapy, v. 54, n. 8, p. 3132-3142, 2010.

HO, C. L. et al. Bamboo Vinegar Decreases Inflammatory Mediator Expression and NLRP3 Inflammasome Activation by Inhibiting Reactive Oxygen Species Generation and Protein Kinase C- $\alpha / \delta$ Activation. PLoS ONE, v. 8, n. 10, 2013.

IBGE, I. B. DE G. E E.-. Produção da extração vegetal e silvicultura. Produção de Extração Vegetal e da Silvicultura, v. 31, n. Rio de Janeiro, p. 55, 2016.

ITÔ, H.; HINO, T. Dwarf bamboo as an ecological filter for forest regeneration. Ecological Research, v. 22, n. 4 , p. 706-711, 2007.

JANKOWSKY, L. et al. Use of Solid Timber Waste as Potential Raw Material for Novel Herbal Drugs : Use of 
Solid Timber Waste as Potential Raw Material for Novel Herbal Drugs : Multidisciplinary Research, Development , and Innovation. v. 1992, n. October, p. 3-8, 2017.

JANKOWSKY, L. et al. Antimicrobial Activity of the Methanolic Fraction of Bamboo Pyroligneous Liquor. Journal of Pharmacy and Pharmacology, v. 6, n. 10, p. 924-934, 2018.

KAN, T.; STREZOV, V.; EVANS, T. J. Lignocellulosic biomass pyrolysis: A review of product properties and effects of pyrolysis parameters. Renewable and Sustainable Energy Reviews, n. 57, p. 1126-1140, 2016.

KESKIN, C. Medicinal Plants and their Traditional Uses. Journal of Advances in Plant Biology, v. 1, n. 2, p. 8$12,2018$.

KOCHAN, K. et al. In vivo atomic force microscopy-infrared spectroscopy of bacteria. Journal of the Royal Society Interface, v. 15, n. 140, 2018.

KRAKER, M. E. A. et al. Mortality and Hospital Stay Associated with Resistant Staphylococcus aureus and Escherichia coliBacteremia: Estimating the Burden of Antibiotic Resistance in Europe. PLoS ONE, v. 8, n. 10, p. e1001104, 2011.

LEONTIA, M.; STICHERA, O.; HEINRICHB, M. Antiquity of medicinal plant usage in two Macro-Mayan ethnic groups (México),. Journal of Ethnopharmacology, v. 88, n. 2-3', p. 119-124, 2003.

LEPAGE, E. S. et al. Manual de Preservação de Madeiras. São Paulo: Instituto de Pesquisas Tecnológicas do Estado de São Paulo - IPT, 1986.

LEVINSON, W. Microbiologia Médica e Imunologia. 16 edt. ed. New Your: Artmed, 2011.

LI, R. et al. Antiviral Activity of Phenolic Derivatives in Pyroligneous Acid from Hardwood, Softwood, and Bamboo. ACS Sustainable Chemistry and Engineering, v. 6, n. 1, p. 119-126, 2018a.

LI, R. et al. Structure-dependent antiviral activity of catechol derivatives in pyroligneous acid against the encephalomycarditis virus. RSC Advances, v. 8, n. 63, p. 35888-35896, 2018b.

LINI, R. S. et al. Caracterização de fármacos por cromatografia em camada delgada. Revista Brasileira de Farmácia, v. 95, n. 1, p. 485-498, 2014.

LOO, A. Y.; JAIN, K.; DARAH, I. Antioxidant activity of compounds isolated from the pyroligneous acid, Rhizophora apiculata. Food Chemistry, v. 107, n. 3, p. 1151-1160, 2008.

MACDOUGALL, C. et al. Hospital and Community Fluoroquinolone Use and Resistance in Staphylococcus aureus and Escherichia coli in 17 US Hospitals. Clinical Infectious Diseases, v. 41, p. 435-440, 2005.

MAHDAVI, M.; CLOUSTON, P.; ARWADE, S. Development of laminated bamboo lumber: review of processing, performance, and econimical considerations. Journal of Materials in Civil Engineering, v. 23, n. 7, p. 1036-1042, 2011.

MALANOVIC, N.; LOHNER, K. Gram-positive bacterial cell envelopes: The impact on the activity of antimicrobial peptides. Biochimica et Biophysica Acta - Biomembranes, p. 936-946, 2016.

MANSUR, D. et al. Production of ketones from pyroligneous acid of woody biomass pyrolysis over an iron-oxide catalyst. Fuel, v. 103, p. 130-134, 2011.

MAPA. Instrução Normativa No 46, De 22 De Novembro De 2016., 2016. Disponível em: <http://www.agricultura.gov.br/assuntos/insumos-agropecuarios/insumos-agricolas/fertilizantes/legislacao/in-46de-22-11-2016-fert-minerais-dou-7-12-16.pdf>

MAPA. Anexo III - Aditivos Autorizados Para Uso em Fertilizantes Minerais, 2017.

MARINHO, N. P. et al. Dendrocalamus giganteus ). Ciência Florestal, v. 22, n. 2, p. 413-418, 2012. 
MARINHO, N. P.; NISGOSKI, S.; DE MUÑIZ, G. I. B. Avaliação das dimensões das fibras de colmos de bambu, Dendrocalamus giganteus (Wall) munro, em diferentes idades. Ciencia Florestal, v. 24, n. 1, p. 251-256, 2014.

MARUMOTO, S. et al. Identification of a germicidal compound against picornavirus in bamboo pyroligneous acid. Journal of Agricultural and Food Chemistry, v. 60, n. 36, p. 9106-9111, 2012.

MATHEW, S.; ZAKARIA, Z. A. Pyroligneous acid-the smoky acidic liquid from plant biomass. Applied Microbiology and Biotechnology, v. 99, n. 2, p. 611-622, 2014.

MENA, L. E. H. Estudo Experimental da Pirólise lenta de Bambu (Dendrocalamus giganteus Munro) e Caracterização do Carvão Vegetal. [s.1.] Universidade Estadual de Campinas - Unicamp, 2014.

MILLER, J. S. The discovery of medicines from plants: A current biological perspective. Econimic Botany, v. 64, n. 4, p. 396-307, 2011.

MOGNON, F. et al. Alocação e modelagem da biomassa em: Dendrocalamus asper. Floresta, v. 45, n. 1, p. 1-10, 2015.

\section{MOKUSAKU. PROVEN EFFECTS OF WOOD VINEGAR TO WOOD VINEGAR.}

MOTA, F. DE A. DA S. et al. Pirólise Da Biomassa Lignocelulósica: Uma Revisão Pyrolysis of Lignocellulose Biomass: a Review. Revista Geintec, v. 5, n. 4, p. 2511-2525, 2015.

MULYONO, N. et al. Antibacterial activity of petung bamboo (Dendrocalamus asper) leaf extract against pathogenic Escherichia coli and their chemical identification. International Journal of Pharmaceutical and Biological Archives, v. 3, n. 4, p. 770-778, 2012.

NOPRIARDY, F.; MULYADI, H.; SETIAJI, B. Liquid Smoke: Rebung Preservation in Canned Food for ASEAN Food Marketing. KnE Social Sciences, v. 3, n. 5, p. 239, 2019.

OLIVEIRA, A. C. et al. Análise de risco econômico de dois sistemas produtivos de carvão vegetal. Floresta e Ambiente, v. 24, n. May, 2017.

OSTROSKY, E. A. et al. Métodos para avaliação da atividade antimicrobiana e determinação da concentração mínima inibitória (CMI) de plantas medicinais. Brazilian Journal of Pharmacognosy, v. 18, n. 2, p. 301-307, 2008.

PEREIRA, M. A. DOS R.; BERALDO, A. L. Bambu de corpo e alma. 2. ed. Bauru, Sâo Paulo: Canal6, 2016.

PETENÁ, G. et al. Scanning electron microscopy of leaf and petal cuts of citrus trees fertigated with two nitrogen sources. Citrus Research \& Technology, v. 37, n. 2, p. 218-225, 2016.

PETTY, N. K. et al. Global dissemination of a multidrug resistant Escherichia coli clone. Proceedings of the National Academy of Sciences, v. 111, n. 15, p. 5694-5699, 2014.

QUEIROZ, M. B.; FERREIRA, M.; RAMBO, M. Correlação entre o poder calorífico e a composição química de biomassas lignocelulósicas . Sociedade Brasileira de Química, n. Ic, p. 1, 2013.

RABELO, C. B. Estudo de caso do consumo de energia verde em uma multinacional do ramo de cosméticos. [s.l.] Universidade Federal do Rio de Janeiro, 2018.

RAMBO, M. K. D. et al. Estudo de análise termogravimétrica de diferentes biomassas lignocelulósicas utilizando a análise por componentes principais. Ciência e Natura, v. 37, n. 3, p. 862-868, 2015.

ROSA, R. A. Caracterização Do Bambu Laminado Colado Como Alternativa Tecnológica Industrial. [s.l.] Universidade Federal do Espírito Santo, 2013.

SANTOS, A. L. et al. Staphylococcus aureus: visitando uma cepa de importância hospitalar. Jornal Brasileiros de Patologia e Medicinal Laboratorial, v. 43, n. 6, p. 413-423, 2007. 
SANTOS, D. R. DE S. et al. Potencial de espécies de Bambu como fonte energética. Scientia Forestalis/Forest Sciences, v. 44, n. 111, p. 751-758, 2016.

SCOZZAFAVA, A. et al. Carbonic anhydrase inhibitors: Guaiacol and catechol derivatives effectively inhibit certain human carbonic anhydrase isoenzymes (hCA I, II, IX and XII). Journal of Enzyme Inhibition and Medicinal Chemistry, v. 30, n. 4, p. 586-591, 2015.

SEN, S.; CHAKRABORTY, R. Revival, modernization and integration of Indian traditional herbal medicine in clinical practice: Importance, challenges and future. Journal of Traditional and Complementary Medicine, v. 7, n. 2, p. 234-244, 2017.

SENA, M. F. M. DE et al. Potencialidades do extrato pirolenhoso: práticas de caracterização. Revista Eletronica em Gestão, Educação e Tecnologia Ambiental - REGET, v. 18, p. 41-44, 2014.

SILVA, M. A relação entre o hábito e a composição da parede celular de bambus nativos. [s.1.] Instituto de Botânica da Secretaria do Meio Ambiente, 2017.

SILVA NETO, J. M. et al. Potencial de Fibras Lignocelulósicas para a Produção de Etanol de Segunda Geração.

5. Encontro Regional de Química e 4. Encontro Nacional de Química, v. 3, n. 1, p. 1038-1047, Nov. 2015.

SNIF. Os Biomas e Suas Florestas - Sistema Nacional de Informações Florestais. Sistema Nacional de Informações Florestais, p. 1-9, 2016.

SOARES, S. G. DE S. C. et al. Characterization of the infections related to health care in a teaching hospital in the Northeastern of Brazil. Revista de Enfermagem da UFPI, v. 6, n. 2, p. 37-43, 2017.

SOUZA, A. P. C. C. Bambu na habitação de interesse social no Brasil. Cadernos de Arquitetura e Urbanismo, v. 11, n. 12, p. 217-245, 2004.

SOUZA, J. L. S. DE et al. Antimicrobial potential of pyroligneous extracts - a systematic review and technological prospecting. Brazilian Journal of Microbiology, v. 49, p. 128-139, 2018.

SOUZA, J. B. G.; RÉ-POPPI, N.; RAPOSO, J. L. Characterization of pyroligneous acid used in agriculture by gas chromatography-mass spectrometry. Journal of the Brazilian Chemical Society, v. 23, n. 4, p. 610-617, 2012.

SOUZA, V. M. A questão da toxicidade nos cosméticos e o aumento dos consumo de cosméticos infantis. Aditivos Cosméticos, 2014.

STOESSER, N. et al. Genomic epidemiology of global Klebsiella pneumoniae carbapenemase (KPC)-producing Escherichia coli. Scientific Reports, v. 7, n. 1, p. 1-11, 2017.

SUMANATRAKUL, P. et al. Utilization of Dendrocalamus Asper Backer Bamboo Charcoal and Pyroligneous Acid. Energy Procedia, v. 79, p. 691-696, 2015.

TAYEL, A. A. et al. Bioactivity and application of plant seeds' extracts to fight resistant strains of Staphylococcus aureus. Annals of Agricultural Sciences, v. 63, n. 1, p. 47-53, 2018.

THEAPPARAT, Y. et al. Physicochemical Characteristics of Wood Vinegars from Carbonization of Leucaena leucocephala, Azadirachta indica, Eucalyptus camaldulensis, Hevea brasiliensis and Dendrocalamus asper. Kasetsart J. (Nat. Sci), v. 48, p. 916-928, 2014.

THEAPPARAT, Y. et al. Pyroligneous acids from carbonisation of wood and bamboo: Their components and antifungal activity. Journal of Tropical Forest Science, v. 27, n. 4, p. 517-526, 2015.

THEAPPARAT, Y.; PONGLIMANONT, C.; CHANDUMPAI, A. In vitro Antioxidant Evaluation of Wood Vinegars from Carbonization of Wood and Bamboo. v. 45, n. 2, p. 868-880, 2018.

TOMBOLATO, A. F. C.; GRECO, T. M.; PINTO, M. M. Dez espécies de bambus exóticos mais comuns no 
paisagismo no Brasil. Revista Brasileira de Horticultura Ornamental, v. 18, n. 2, p. 105-114, 2012.

TORTORA, G. J.; FUNKE, B. R.; CASE, C. L. Microbiologia. 8. ed. Porto Alegre: Artmed, 2005.

VIEIRA, G. E. G. et al. Biomassa: uma visão dos processos de pirólise. Revista Liberato, v. 15, n. 24, p. 168$177,2014$.

VIEIRA, P. N.; VICENTINO VIEIRA, S. L. Uso Irracional E Resistência a Antimicrobianos Em Hospitais. Arquivos de Ciências da Saúde da UNIPAR, v. 21, n. 3, p. 209-212, 2018.

WEINSTEIN, M. P. et al. Performance Standards for Antimicrobial Susceptibility Testing - ED28/2018.

Disponível em: <http://em100.edaptivedocs.net/GetDoc.aspx?doc=CLSI M100 ED28:2018\&scope=user >. Acesso em: 25 jun. 2018.

YALEW, R. et al. Antibacterial effects of the tellurium compound OTD on E. coli isolates. Archives of Microbiology, v. 196, n. 1, p. 51-61, 2014.

YU, X. Bamboo: Structure and Culture. p. 196, 2008.

ZANETTI, M. et al. Uso de subprodutos de carvão vegetal na formação do porta-enxerto limoeiro «cravo» em ambiente protegido. Rev. Bras. Frutic., v. 25, n. 3, p. 508-512, 2003.

ZETOLA, N. et al. Community-acquired meticillin-resistant Staphylococcus aureus: an emerging threat Nicola. Lancet Infect Dis, v. 5, p. 15275-286, 2005.

ZHAO, D. et al. Intracellular antioxidant effect of vanillin, 4-methylguaiacol and 4-ethylguaiacol: Three components in Chinese Baijiu. RSC Advances, v. 7, n. 73, p. 46395-46405, 2017.

ZHOU, Y. K.; SHEN, X. Y.; HOU, C. L. Diversity and antimicrobial activity of culturable fungi from fishscale bamboo (Phyllostachys heteroclada) in China. World Journal of Microbiology and Biotechnology, v. 33, n. 6, p. 1-7, 2017. 